\title{
A Robust Watermarking Scheme Based on Maximum Wavelet Coefficient Modification and Optimal Threshold Technique
}

\author{
Chunlei Li, Xiaowei Song, Zhoufeng Liu, Aihua Zhang, and Ruimin Yang \\ School of Electronic and Information Engineering, Zhongyuan University of Technology, Zhengzhou 450007, China \\ Correspondence should be addressed to Chunlei Li; lichunlei1979@sina.com
}

Received 15 April 2015; Revised 12 June 2015; Accepted 16 July 2015

Academic Editor: René Cumplido

Copyright (C) 2015 Chunlei Li et al. This is an open access article distributed under the Creative Commons Attribution License, which permits unrestricted use, distribution, and reproduction in any medium, provided the original work is properly cited.

\begin{abstract}
Digital watermarking has received extensive attention as a new method for copyright protection. This paper proposes a robust watermarking algorithm based on maximum wavelet coefficient modification and optimal threshold technique. The medium wavelet coefficients are randomly permutated according to a secret key and divided into subgroups. We modify the maximum wavelet in each subgroup according to the embedded watermark bits, which can effectively resist attacks. During the extraction process, an optimal threshold value calculated by iterative computation is used to extract the watermark from the watermarked image under different attacks, without using the original image or watermark. Numerous experiments are conducted to evaluate the watermarking performance. Experimental results demonstrate the superiority of our scheme on robustness against contentpreserving operations and incidental distortions such as JPEG compression, Gaussian noise, median filter, resizing, cropping, and sharpening.
\end{abstract}

\section{Introduction}

Currently, with rapid growth of the Internet and digital media, large amounts of media data are transmitted through the Internet due to its convenience and amazing speed. Digital media content and copyright are confronted with great challenges. Many researchers are aware of the importance of copyright protection, image authentication, and so forth, and they have great interest in applying watermarking scheme into digital multimedia for copyright protection $[1,2]$. One of the most important branches in digital watermarking community is robust watermarking, which aims at achieving robustness, imperceptibility, and high security simultaneously [3-7].

However, the robustness and imperceptibility are contradictory to each other, and thus a good embedding scheme is required to achieve an appropriate trade-off between them. Basically, the watermark can be embedded in either the spatial domain or the transform domain. The former embeds a watermark into the host image by directly modifying the pixel value of the host image [8-10]. In contrast, the latter firstly performs the domain transformation and then embeds watermarks by modifying the coefficients in transform domain. In general, watermarking in transform domain is more robust than the one in spatial domain. In the past decades, a lot of watermarking algorithms have been developed in transform domain, for example, discrete cosine transforms (DCT) [11] and discrete wavelet transforms (DWT) [12].

Comparing DWT for JPEG2000 with DCT for JPEG, DWT has merits such as no blockiness, fast processing time, and high compression ability [13]; the robust watermarking scheme based on DWT has attracted great interest.

Wavelet-based watermarking scheme can be classified into two categories: wavelet tree-based watermarking methods and block-based DWT watermarking methods. The wavelet tree-based watermarking methods are generally using the energy difference among grouped wavelet coefficients for invisible watermark embedding and extraction [14-22]. Wang and Lin [14] grouped two wavelet trees into a so-called supertree, and each bit is embedded into two supertrees. The two trees exhibit a large statistical difference after one of the two trees is quantized with respect to a quantization index, which will be used for watermark extraction. Lien and Lin [15] improved Wang's method by using four trees to represent two watermark bits in order to 
improve visual quality. Wu and Huang [16] embedded the watermark into the supertrees by structure-based quantization method. According to watermark bits, the supertrees will be quantized into a significant structure. Compared to the unquantized supertree, the quantized version has strong statistical character in energy distribution, which can be used to extract watermark bits. In [19], wavelet trees are classified into two clusters using the distance vector to denote binary watermark bits and the two clusters exhibit a sufficiently large statistical difference based on the distance vector, a difference which is then used for subsequent watermark extraction. Tsai [21] enhanced the security of wavelet tree quantization watermarking scheme by adopting the chaotic system. Run et al. [22] embedded a watermark bit in the maximum wavelet coefficient of a wavelet; this is different from those in [1416] which use two trees to embed a watermark bit. And the embedding method modifies the magnitude of the significant difference between the two largest wavelet coefficients in a wavelet tree to improve the robustness of the watermarking.

On the other hand, some researches embed a watermark using block-based DWT [23-31]. Davoine [23] proposed the watermarking methods based on the triplets and rectangular blocks of significant wavelet coefficients. Zhang et al. [24] divided the original image into blocks and transformed them into a DWT domain. The watermark is embedded by using the mean and the variance of a subband to modify the wavelet coefficient of a block. Khelifi et al. [25] proposed an adaptive blind watermarking method based on DWT. The host image is separated into nonoverlapping blocks classified as uniform or nonuniform blocks using a JND-based classifier. The watermark is embedded in the high subband of each block according to its classification. In [26-28], the block-based watermarking in the wavelet domain is proposed. They applied the significant difference between the first and second greatest coefficients to distinguish the bipolar watermark. Verma and Jha [29] Improved significant difference-based watermarking technique using lifting wavelet coefficients. In [30], the embedding algorithm hides a watermark bit in the low-low (LL) subband of a target nonoverlap block of the host image by modifying a coefficient of $U$ component on SVD version of the block. A blind watermark extraction is designed using a trained SVR to estimate original coefficients. Subsequently, the watermark bit can be computed using the watermarked coefficient and its corresponding estimate coefficient. Sahraee and Ghofrani [31] propose a robust blind watermarking algorithm based on quantization of distance among wavelet coefficients for copyright protection. The authors divided wavelet coefficients into some blocks and obtained the first, second, and third maximum coefficients in each block. Then, the first and second maximum coefficients are quantized according to binary watermark bits. Using the block-based watermarking, the watermark can be extracted without using the original image or watermark.

The above-mentioned methods focused on locating the significant DWT component as embedding candidates and formulate appropriate strategy to modulate them without raising perceptual distortion. However, watermark extraction scheme is also critical for watermarking methods. In this paper, we propose a robust watermarking scheme based on maximum wavelet coefficient modification and optimal threshold technique. The medium wavelet coefficients are randomly permutated and divided into nonoverlapped groups based on a private key. In each group, the largest two coefficients in a block are called significant coefficients and their difference is called significant difference. We modify the maximum wavelet coefficients to guarantee that the significant difference between watermark bit 0 and watermark bit 1 exhibits a large energy difference. During the extraction process, an optimal threshold value calculated by iterative computation is used to extract the watermark from the watermarked image under different attacks, without using the original image or watermark.

The remainder of the paper is organized as follows. In Section 2, we give an overview of DWT significant difference quantization based watermarking methods proposed by Lin et al. [26]; in Section 3, the details of our proposed robust watermarking algorithms are given; in Section 4, the experimental results and analysis are presented; and finally Section 5 concludes the proposed scheme.

\section{Watermarking Method Based on Significant Difference of Wavelet Coefficient Quantization}

Locating the significant coefficients embedding and adopting appropriate strategy to modulate them without raising perceptual distortion are two critical issues for DWT based watermarking methods. Compared with previous work, the methods based on group significant wavelet coefficients proposed by Lin et al. [26] can blindly find the permutation of significant coefficients and modulate the maximum to provide good watermarking robustness. The proposed method can be described as follows.

The proposed method in Lin et al. [26] firstly transforms the host image into wavelet domain by 3 -L wavelet transform (DWT). Then LH3 which is more significant than HL3 is used for embedding watermarks. Every seven consecutive coefficients in LH3 subband are grouped into a block, and a secret key is then utilized to randomly select $N_{w}$ blocks to embed the watermark bits $W=\left\{w_{k} \in\{0,1\} \mid k=1,2, \ldots, N_{w}\right\}$. The quantization method is to adjust the significant difference (the difference between the first and second largest coefficients) to represent the binary watermark. If the embedding watermark bit is 1 , then $\max _{k}$ is modified as $\max _{k}^{\prime}$ as:

$$
\begin{aligned}
& \max _{k}^{\prime} \\
& \quad= \begin{cases}\max _{k}+Q, & \text { if }\left(\max _{k}-\sec _{k}\right)<\operatorname{maxium}(\varepsilon, Q) \\
\max _{k}, & \text { otherwise, }\end{cases}
\end{aligned}
$$

where $\max _{k}$ is the largest coefficient and $\sec _{k}$ is the second largest coefficient in the $k$ th block. $\varepsilon$ is the average significant difference of all $N_{w}$ blocks and $Q$ is the embedding strength. Otherwise, if the watermark bit is 0 , the embedding equation is described as

$$
\max _{k}^{\prime}=\sec _{k}
$$




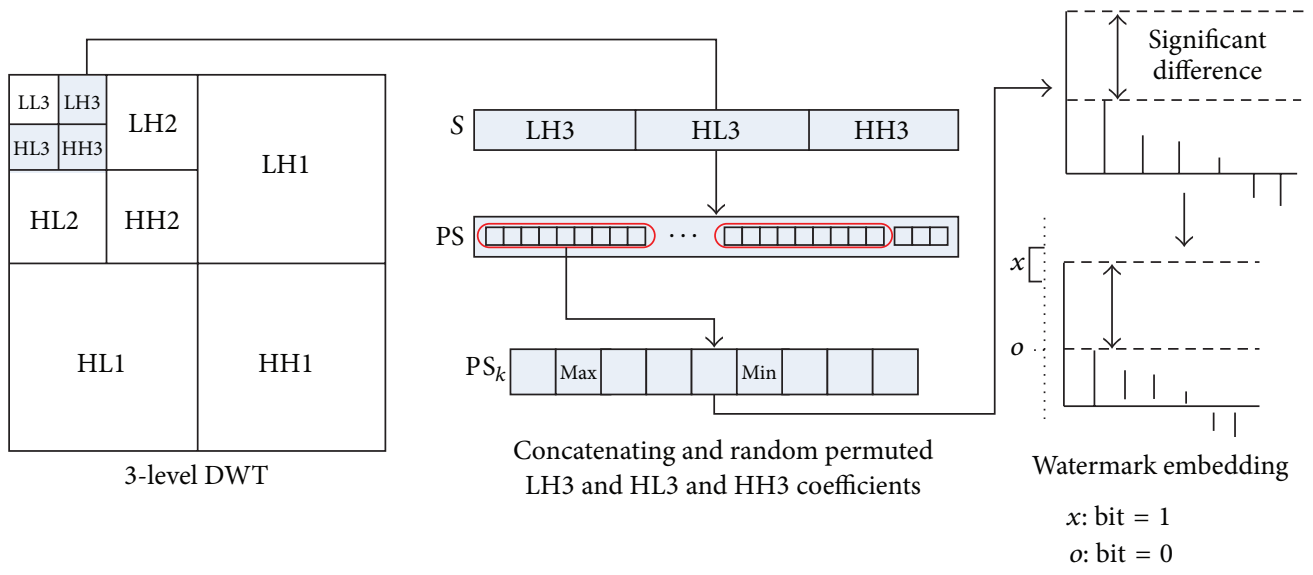

FIGURE 1: Watermark bits embedding.

The difference between watermark bits " 0 " and " 1 " is significant after embedding. In order to extract watermark bits $W$, a statistic difference is analyzed by (3) to find a threshold $\tau$; it depends on the ratio between two watermark symbols:

$$
\tau=\left\lfloor\frac{1}{N_{w} \alpha} \sum_{j=1}^{N_{w} \alpha} \delta_{j}\right\rfloor,
$$

where $\delta_{1} \leq \delta_{2} \leq \cdots \leq \delta_{j}$ are ordered significant difference and $\alpha(0<\alpha \leq 1)$ is an empirical trade-off parameter and is sensitive to the ratio of two kinds of binary watermark bits. When the two kinds of binary watermark bits are equiprobable, $\alpha$ is set to 0.9 .

Next, watermark information bits $w_{k}$ are extracted by comparing the significant difference with the threshold $\tau$, and it can be described as follows:

$$
w_{i}= \begin{cases}1, & \text { if }\left(\max _{k}^{\prime}-\sec _{k}^{\prime}\right) \geq \tau \\ 0, & \text { otherwise. }\end{cases}
$$

The normalized correlation coefficient (NC) is calculated using the original watermark $w_{i}$ and the extracted watermark $w_{i}^{\prime}$ to judge the existence of watermark, and it can be defined as follows:

$$
\mathrm{NC}\left(W, W^{\prime}\right)=\frac{1}{N_{w}} \sum_{j=1}^{N_{w}} w_{j} w_{j}^{\prime} .
$$

The value is compared with a threshold value $\rho$. If $\mathrm{NC} \geq \rho$, and it demonstrated that the extracted watermark is existing [26]; otherwise, it does not. When $N_{w}$ is 512, the false positive error is $1.03 \times 10^{-7}$ and $\rho$ is set to 0.23 .

\section{The Proposed Robust Watermarking Scheme}

In Lin et al. [26], each watermark bit is embedded into a block consisting of consecutive seven coefficients of HL3 subband. However, Meerwald et al. [33] pointed out that an attacker can analyze the property of embedding method and destroy the watermark. In this paper, the coefficients are selected from three subbands (HL3, LH3, and HH3) and then grouped into blocks after random permutation. A watermark bit is embedded into a coefficient group by improving maximum wavelet coefficient modification. Moreover, both the watermarked image quality and security can be achieved simultaneously. Finally, watermark extraction can be converted to threshold segmentation, and then an optimal threshold calculated by iterative computation is adopted to efficiently extract watermarks.

3.1. Watermark Embedding. In order to improve security of the proposed method, a random sequence is generated by utilizing the logistic chaotic map to encrypt the generated watermarks $W=\left\{w_{i}, i=1,2, \ldots, N_{w}\right\}$ [34]:

$$
y_{n+1}=\lambda y_{n}\left(1-y_{n}\right) \text {, }
$$

where $3.57<\lambda \leq 4$ and $0<y_{0} \leq 0.5$. This sequence is nonperiodic, nonconvergent, and very sensitive to the initial value $y_{0}$; thus, the secret key $k_{1}$ is formulated as follows: $k_{1}=\left\{\lambda, y_{0}\right\}$. We set $\lambda$ and $y_{0}$ to 3.78 and 0.43 , respectively. Then we binarize this sequence to a binary string $s_{j}(j=$ $\left.1,2, \ldots, N_{w}\right) \in\{0,1\}$. Exclusive-OR operation is used to encrypt information bits by using

$$
e w_{i}=w_{i} \oplus s_{i}, \quad i=1,2, \ldots, N_{w} .
$$

Figure 1 illustrates the watermark embedding process. We firstly apply three-level wavelet decomposition into the original host image with size of $512 \times 512$ and select LH3, HL3, and $\mathrm{HH} 3$ subbands as embedding candidates. Three groups of $64 \times 64$ midfrequency coefficients are converted into 4096-dimension sequences, respectively. Thereafter, we concatenate the sequences into a single sequence $S$ with 12288 coefficients. To enhance the security of the system, the sequence $S$ is permuted as PS by using the secret key $k_{2}$. The permuted sequence PS is divided into $N_{w}$ subgroups denoted as PS $=\mathrm{PS}_{k}, k=1,2, \ldots, N_{w}$, where $N_{w}$ is the number of watermark bits. We embed one bit for each group.

Dither modulation scheme is an improvement of the original uniform QIM (quantization index modulation) algorithm proposed by Chen and Wornell [35]. Applying dither 
modulation in watermark embedding process can tackle the quantization noise; it has two advantages: first, a pseudorandom dither vector can reduce quantization artifacts and get a perceptually superior quantized content. Second, the private key based dither vector can be secretly shared by the embedder and extractor and thus improve the system security.

Therefore, we improve the embedding method in Lin et al. [26] by adopting dither modulation scheme. And the embedding process can be described as follows:

(1) Generate the pseudorandom dither vectors $d\left[k, w_{k}\right]$ according to another private key $k_{3} . d[k, 0]$ is randomly generated from $[-Q / 4, Q / 4]$, and $d[k, 1]$ is generated using the following equation:

$$
d[k, 1]= \begin{cases}d[k, 0]+\frac{Q}{4}, & d[k, 0] \geq 0 \\ d[k, 0]-\frac{Q}{4}, & \text { otherwise, }\end{cases}
$$

where $T$ is embedding strength set by embedder.

(2) Embed the watermark bit by modifying the maximum wavelet in each subgroup. If a watermark bit 1 is embedded, we quantize $\max _{i}$ with $Q$ and dither vectors $d[k, 1]$ as shown in (7):

$$
\max _{i}^{\text {new }}= \begin{cases}\sec _{i}+Q+d[k, 1], & \text { if } \max _{i}-\sec _{i}<\mathrm{Q} \\ \max _{i}+d[k, 1], & \text { otherwise, }\end{cases}
$$

where $\sec _{i}$ is the second largest coefficient, $\max _{i}$ is the largest coefficient in subgroup $\mathrm{PS}_{i}$, and $\max _{i}^{\text {new }}$ is denoted as the new maximum value. If the difference between $\max _{i}$ and $\sec _{i}$ is smaller, we need an extra parameter $Q$ and $d[k, 1]$ to do quantization. Otherwise, the difference is obvious and we only use $d[k, 1]$ to do quantization.

On the other hand, if a block is embedded with a watermark bit $0, \max _{i}$ is quantized as follows:

$$
\max _{i}^{\text {new }}=\sec _{i}+d[k, 0] .
$$

Finally, we recover the watermarked coefficients to original positions by $k_{2}$ and apply inverse wavelet transform to obtain the final watermarked image.

\section{Algorithm 1 (watermark embedding).}

Input. It includes an original image and watermark $W$.

Output. It includes a watermarked image with size of $512 \times$ 512:

(1) Encrypt watermarks using (7).

(2) Apply three-level wavelet decomposition to the host image and select LH3, HL3, and HH3 subbands as embedding candidates.

(3) Concatenate the three subbands coefficient sequences into a single sequence $S$, and then randomly permute $S$ using secret key $k_{2}$ to get PS.

(4) Equally split PS into $N_{w}$ coefficient groups $\mathrm{PS}_{i}$.
(5) Generate the dither vectors $d[k, 0]$ and $d[k, 1]$ using (8).

(6) Embed the watermark bit $w_{i}$ into the $i$ th group, $i=$ $1,2, \ldots, N_{w}$ using (9) or (10).

(7) Recover the watermarked coefficients, and apply inverse wavelet transform to get the watermarked image.

3.2. Watermark Extraction. In the proposed method, either an original image or an original watermark is not required for the extraction process. The difference between the largest coefficient and the second coefficient is used to extract watermark. If watermarked image keep unaltered, the difference is less than $Q / 4$ for embedding " 0 "; on the other hand, the difference is above $3 / 4 Q$ for embedding " 1 ." Therefore, watermark extraction can be converted into threshold segmentation. For a coefficient group, when the significant difference is satisfied with $d_{i}>T$, where $d_{i}=\left(\max _{i}^{\prime}-\right.$ $\sec _{i}^{\prime}$ ); there is a large probability that a watermark bit 1 was embedded; otherwise, a watermark bit 0 was embedded, where $T$ is an optimal threshold obtained by iterations.

First, the differences are segmented into two parts. Then the mean value of the two parts is, respectively, computed. The average of the two mean values is taken as an updated threshold to iteratively segment the differences until the process converges. The process can be described as follows.

(1) Calculate the initial threshold $T_{0}(k=0)$ by

$$
T_{0}=\frac{d_{\min }+d_{\max }}{2}
$$

where $d_{\min }$ and $d_{\max }$ are, respectively, the minimum and the maximum value of the differences.

(2) Split the differences via threshold $T_{k}(k=0,1, \ldots$, into two parts of $R_{1}$ and $R_{2}$, and it can be written as

$$
\begin{aligned}
& R_{1}=\left\{d_{i} \mid d_{i} \geq T_{k}\right\}, \\
& R_{2}=\left\{d_{j} \mid 0 \leq d_{j}<T_{k}\right\} .
\end{aligned}
$$

(3) Calculate the mean value of $R_{1}$ and $R_{2}$ :

$$
\begin{aligned}
& Z_{1}=\sum_{i=1}^{N_{L}} \frac{d_{i}}{N_{L}}, \\
& Z_{2}=\sum_{j=1}^{N_{H}} \frac{d_{j}}{N_{H}},
\end{aligned}
$$

$N_{L}$ is the number of the differences which are less than $T_{k}$, and $N_{H}$ is the number of the differences which are larger than $T_{k}$.

(4) Update threshold $T_{k+1}$ :

$$
T_{0}=\frac{Z_{1}+Z_{2}}{2} \text {. }
$$

(5) If $\left|T_{k+1}-T_{k}\right|<\delta$ ( $\delta$ is a predetermined small value), end the iteration; otherwise go to Step (2).

After generating the optimal threshold $T$, the watermark bit can be extracted as

$$
b_{i}= \begin{cases}1, & \text { if }\left(\max _{i}^{\prime}-\sec _{i}^{\prime}\right) \geq T \\ 0, & \text { otherwise }\end{cases}
$$




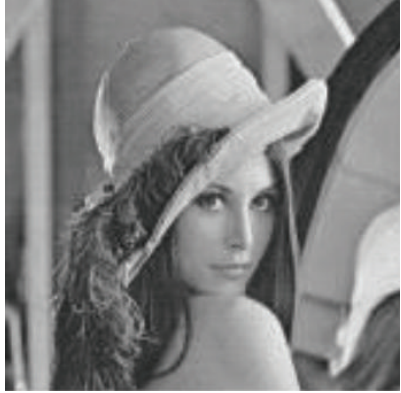

(a)

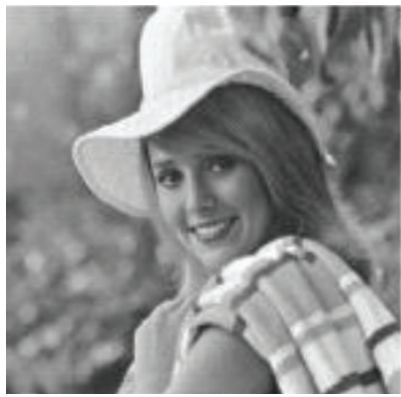

(e)

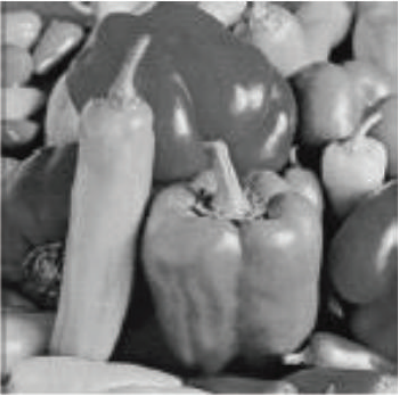

(b)

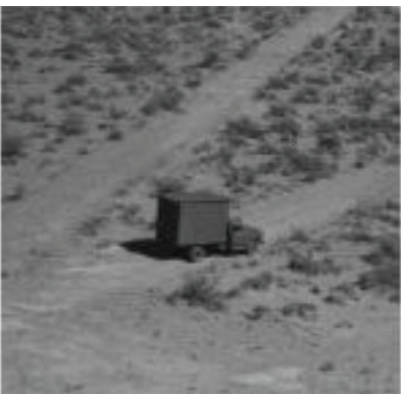

(f)

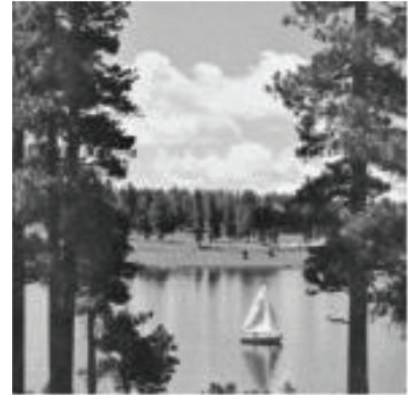

(c)

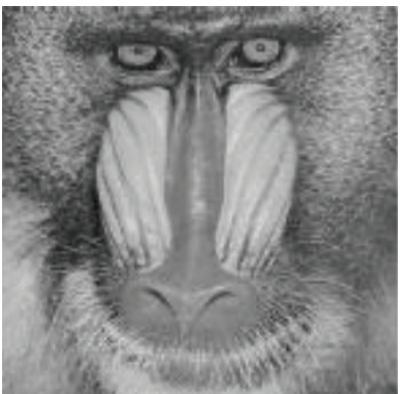

(g)

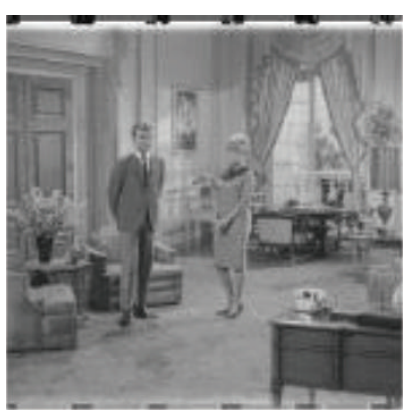

(d)

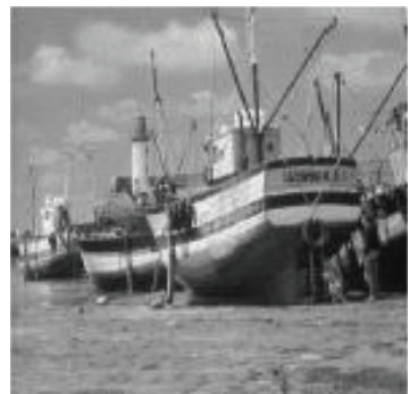

(h)

Figure 2: Selected images used in our experiment. (a) Lena, (b) pepper, (c) sailboat, (d) couple, (e) Elaine, (f) trunk, (g) mandrill, and (h) ship (all images are from USC-SIPI).

Algorithm 2 (watermark extraction).

Input. It includes a watermarked image.

Output. It includes a binary watermark sequence:

(1) A $512 \times 512$ watermarked image is decomposed using the 3-level DWT; select LH3, HL3, and HH3 subbands to extract watermarks.

(2) Concatenate the three subbands coefficient sequences into a single sequence $S$, and then randomly permute $S$ using secret key $k_{2}$ to get PS as we have done in embedding procedure.

(3) Equally split PS into $N_{w}$ coefficient groups $\mathrm{PS}_{i}$.

(4) Get the values of $\max _{i}^{\prime}-\sec _{i}^{\prime}$ for each coefficient group.

(5) Calculate an optimal threshold value using (11)-(14).

(6) Extract the watermark bits using (15).

\section{Experimental Results and Analysis}

Cohen-Daubechies-Fauraue (CDF) 9/7 wavelet is a biorthogonal wavelet and has better energy compaction and lower computational cost, and it is selected as a part of the JPEG2000 standard. Therefore, CDF 9/7 wavelet is used for watermark embedding in our method. Meantime, twenty eight-bit gray scale images of size $512 \times 512$ from the USCSIPI database [36] are used for evaluating watermarking performance, and the selected eight images are presented in Figure 2.
TABLE 1: Embedding strength versus PSNR and SSIM.

\begin{tabular}{lcccccccc}
\hline Q & 10 & 20 & 30 & 40 & 50 & 60 & 70 & 80 \\
\hline PSNR (dB) & 48.75 & 46.57 & 45.18 & 44.23 & 43.19 & 42.17 & 41.81 & 41.79 \\
SSIM & 0.99 & 0.99 & 0.98 & 0.98 & 0.98 & 0.98 & 0.97 & 0.97 \\
\hline
\end{tabular}

For making comparisons with our method and the other watermarking methods $[14,26,27,32]$, random binary sequences of the same length $N_{w}=512$ with equal ratio of "0" and " 1 " are employed as watermarks. Therefore, a watermark bit can be embedded into a coefficient group with size 24 in our method.

4.1. Embedding Distortion Assessment. To measure the embedding distortion between original image and its watermarked image, the peak signal-to-noise ratio (PSNR) and structural similarity index (SSIM) are adopted in this paper, in which, the definition of SSIM is as follows:

$$
\operatorname{SSIM}(x, y)=\frac{\left(2 u_{x} u_{y}+C_{1}\right)\left(2 \sigma_{x y}+C_{2}\right)}{\left(\mu_{x}^{2}+\mu_{y}^{2}+C_{1}\right)\left(\sigma_{x}^{2}+\sigma_{y}^{2}+C_{2}\right)} .
$$

Table 1 presents the PSNR and SSIM for different embedding strength $Q$. By increasing the embedding strength, imperceptibility decreases. Thus, a trade-off is made in selecting the suitable quantization step. Generally, the PSNR values are above $40 \mathrm{~dB}$ often which corresponds to almost invisible differences. In this paper, we set the quantization step to 60 , PNSR is up to $42 \mathrm{~dB}$, and SSIM is 0.98 . 
TABLE 2: Embedding strength versus PSNR and SSIM.

\begin{tabular}{lccccccccc}
\hline & 10 & 15 & 20 & 25 & 30 & 40 & 50 & 70 & 90 \\
\hline WTQ [14] (39.6 dB) & - & - & - & - & 0.24 & 0.37 & 0.49 & 0.75 & 1 \\
SDWCQ [26] (42.2 dB) & 0.42 & 0.56 & 0.70 & 0.80 & 0.86 & 0.94 & 0.97 & 1 & 1 \\
MWCQ [27] (41.5 dB) & 0.35 & 0.52 & 0.69 & 0.81 & 0.85 & 0.92 & 0.95 & 0.96 & 0.99 \\
SD-QIM [32] (43.3 dB) & 0.39 & 0.72 & 0.91 & 0.96 & 0.98 & 0.99 & 1 & 1 & 1 \\
Our method (42.2 dB) & 0.61 & 0.84 & 0.96 & 0.99 & 0.99 & 1 & 1 & 1 \\
\hline
\end{tabular}

TABLE 3: Comparisons of robustness against JPEG2000 compression.

\begin{tabular}{lccccccccc}
\hline & 0.1 & 0.125 & 0.15 & 0.2 & 0.25 & 0.3 & 0.4 & 0.5 & 0.6 \\
\hline WTQ [14] (39.6 dB) & - & - & - & - & - & 0.25 & 0.36 & 0.48 & 0.61 \\
SD-QIM [32] (43.3 dB) & 0.35 & 0.56 & 0.61 & 0.63 & 0.84 & 0.99 & 0.99 & 0.99 & 1 \\
Our method (42.2 dB) & 0.59 & 0.88 & 0.93 & 0.96 & 0.99 & 0.99 & 1 & 1 & 1 \\
\hline
\end{tabular}

TABLE 4: Comparisons of robustness against incidental manipulation.

\begin{tabular}{|c|c|c|c|c|c|c|}
\hline & Media $(3 \times 3)$ & Media $(5 \times 5)$ & Resizing (0.5) & Cropping (25\%) & Sharpening & $\overline{\text { Gauss }}$ \\
\hline WTQ [14] (39.6 dB) & 0.51 & - & - & - & 0.46 & 0.64 \\
\hline SDWCQ [26] (42.2 dB) & 0.88 & 0.74 & 0.86 & 0.70 & 0.99 & 0.86 \\
\hline MWCQ [27] (41.5 dB) & 0.90 & 0.76 & 0.88 & 0.66 & 0.97 & 0.88 \\
\hline SD-QIM [32] (43.3 dB) & 0.94 & 0.42 & 0.98 & 0.87 & 0.21 & 1 \\
\hline Our method (42.2 dB) & 0.96 & 0.62 & 0.93 & 0.91 & 0.91 & 0.99 \\
\hline
\end{tabular}

4.2. Robustness Assessment. To evaluate the robustness quantitatively, normalized correlation coefficient is adopted, and it is described as follows:

$$
\mathrm{NC}\left(W, W^{\prime}\right)=\frac{W \cdot W^{\prime}}{\|W\| \cdot\left\|W^{\prime}\right\|},
$$

where $W$ is the original watermark, $w^{\prime}$ is the extracted watermark, and $\|\cdot\|$ denotes the $\zeta_{2}$ norm. In this equation, if we map watermark " 0 " to " -1 ", we can get $\|W\| \cdot\left\|W^{\prime}\right\|=$ $\sqrt{\sum_{k=1}^{N_{w}} w_{k}^{2} \cdot \sum_{k=1}^{N_{w}}\left(w_{k}^{\prime}\right)^{2}}=N_{w}$. Then, (17) can be rewritten as the following equation:

$$
\mathrm{NC}\left(W, W^{\prime}\right)=\frac{W \cdot W^{\prime}}{N_{w}}=\frac{1}{N_{w}} \sum_{k=1}^{N_{w}} w_{k} \cdot w_{k}^{\prime} .
$$

Thereafter, we evaluate the robustness against the common image processing operations and incidental distortions such as lossy compression and noise. First, the robustness of our method under JPEG compression is compared with other methods. Twenty images are selected in our experiment, and the average value for NC is adopted for comparison. The experimental results are presented in Table 2 . And the image quality metrics and NC values of other methods are collected from the original papers. From Table 2, we can observe that our method has the best robustness while keeping a high fidelity. Nearly all 512 bits can be accurately extracted when the quality factor is up to 25. Even when the QF decrease to 10 , nearly $60 \%$ watermark bits can be correctly extracted, which demonstrates a significant advantage over other methods.
Since our method is designed within the DWT domain, it has natural robustness to the DWT quantization based JPEG2000 compression. And the comparing results are presented in Table 3. From the comparison, we can see that our method has advantages over classical DWT based methods. Next, the robustness against the miscellaneous attacks is presented in Table 4. For the cropping attack, the pixel in the $25 \%$ area of the whole watermark image in the upper left is set to zeros. For the resizing attack, the watermarked images are downsampled by a scale factor 0.5 and afterward interpolated to original resolution for watermark extraction. For the median filter, the $3 \times 3$ and $5 \times 5$ window sizes are used in the experiments. For the sharpening attack, the convolving mask is created from the negative of the Laplacian filter with parameter alpha equal to 0.2. For the Gaussian filter, it is zero mean noise with 0.005 variances. From Table 4, we can conclude that our proposed method is robust to these attacks.

\section{Conclusions}

In this paper, a robust watermarking scheme based on maximum wavelet coefficient modification and optimal threshold technique is proposed. The main advantages are threefold: (1) security is enhanced by randomly permuting coefficients among a group and image robustness is improved by embedding the watermark in the largest coefficient inside a subgroup by significant difference parity quantization; (2) the adopted dither quantization method can efficiently take control of watermarking distortions and correctly extract the watermarks under various attack conditions; (3) an optimal 
threshold value is used to extract the watermark from the watermarked image under different attacks, without using the original image or watermark. Comparison results with WTQ SDWCQ, MWCQ, and SD-QIM methods prove that the proposed method can provide better robustness toward a large variety of attacks while keeping relative high fidelity and capacity. In the future work, introducing HVS models for adaptive quantization of the significant amplitude coefficients is the main focus.

\section{Conflict of Interests}

The authors declare that there is no conflict of interests regarding the publication of this paper.

\section{Acknowledgments}

This work was supported by the National Natural Science Foundation of China (nos. 61202499, 61379113, 61440031, and 60902063), the Project of Henan Provincial Key Science and Technology Research (nos. 142102210578, 132300410163, and 142300410042), and Plan for Scientific Innovation Talent of Henan Province (124100510015).

\section{References}

[1] I. J. Cox, J. Kilian, F. T. Leighton, and T. Shamoon, "Secure spread spectrum watermarking for multimedia," IEEE Transactions on Image Processing, vol. 6, no. 12, pp. 1673-1687, 1997.

[2] W. Zeng and B. Liu, "A statistical watermark detection technique without using original images for resolving rightful ownerships of digital images," IEEE Transactions on Image Processing, vol. 8, no. 11, pp. 1534-1548, 1999.

[3] J.-L. Liu, D.-C. Lou, M.-C. Chang, and H.-K. Tso, "A robust watermarking scheme using self-reference image," Computer Standards \& Interfaces, vol. 28, no. 3, pp. 356-367, 2006.

[4] R. Liu and T. Tan, "An SVD-based watermarking scheme for protecting rightful ownership," IEEE Transactions on Multimedia, vol. 4, no. 1, pp. 121-128, 2002.

[5] L.-T. Ko, J.-E. Chen, H.-C. Hsin, Y.-S. Shieh, and T.-Y. Sung, "Haar-wavelet-based just noticeable distortion model for transparent watermark," Mathematical Problems in Engineering, vol. 2012, Article ID 635738, 14 pages, 2012.

[6] B.-Z. Li and Y.-P. Shi, "Image watermarking in the linear canonical transform domain," Mathematical Problems in Engineering, vol. 2014, Article ID 645059, 9 pages, 2014.

[7] J. Lu, T. Qu, and H. R. Karimi, "Novel iris biometric watermarking based on singular value decomposition and discrete cosine transform," Mathematical Problems in Engineering, vol. 2014, Article ID 926170, 6 pages, 2014.

[8] M. Kutter, F. Jordan, and F. Bossen, "Digital watermarking of color images using amplitude modulation," Journal of Electronic Imaging, vol. 7, no. 2, pp. 326-332, 1998.

[9] M. Kutter and S. Winkler, "A vision-based masking model for spread-spectrum image watermarking," IEEE Transactions on Image Processing, vol. 11, no. 1, pp. 16-25, 2002.

[10] Y.-S. Juang, L.-T. Ko, J.-E. Chen, Y.-S. Shieh, T.-Y. Sung, and H. C. Hsin, "Histogram modification and wavelet transform for high performance watermarking," Mathematical Problems in Engineering, vol. 2012, Article ID 164869, 14 pages, 2012.
[11] M. Barni, F. Bartolini, V. Cappellini, and A. Piva, "A DCTdomain system for robust image watermarking," Signal Processing, vol. 66, no. 3, pp. 357-372, 1998.

[12] D. Kundur and D. Hatzinakos, "A robust digital image watermarking method using wavelet-based fusion," in Proceedings of the International Conference on Image Processing, vol. 3, pp. 544547, 1997.

[13] M. Rabbani and R. Joshi, "An overview of the JPEG 2000 still image compression standard," Signal Processing: Image Communication, vol. 17, no. 1, pp. 3-48, 2002.

[14] S.-H. Wang and Y.-P. Lin, "Wavelet tree quantization for copyright protection watermarking," IEEE Transactions on Image Processing, vol. 13, no. 2, pp. 154-165, 2004.

[15] B. K. Lien and W. H. Lin, "A watermarking method based on maximum distance wavelet tree quantization," in Proceedings of the 19th Conference on Computer Vision, Graphics and Image Processing, 2006.

[16] G.-D. Wu and P.-H. Huang, "Image watermarking using structure based wavelet tree quantization," in Proceedings of the 6th IEEE/ACIS International Conference on Computer and Information Science (ICIS '07), pp. 315-319, IEEE, July 2007.

[17] M.-J. Tsai and C.-L. Lin, "Constrained wavelet tree quantization for image watermarking," in Proceedings of the IEEE International Conference on Communications (ICC '07), pp. 1350-1354, IEEE, June 2007.

[18] M.-J. Tsai, C.-T. Lin, and J. Liu, "A wavelet-based watermarking scheme using double wavelet tree energy modulation," in Proceedings of the 15th IEEE International Conference on Image Processing (ICIP '08), pp. 417-420, IEEE, San Diego, Calif, USA, October 2008

[19] W.-H. Lin, Y.-R. Wang, and S.-J. Horng, "A wavelet-tree-based watermarking method using distance vector of binary cluster," Expert Systems with Applications, vol. 36, no. 6, pp. 9869-9878, 2009.

[20] R. Shijie and S. Xin, "A wavelet-tree-based watermarking method using fast ICA," in Proceedings of the 2nd International Symposium on Computational Intelligence and Design (ISCID '09), vol. 2, pp. 162-164, IEEE, Changsha, China, December 2009.

[21] M. J. Tsai, "Security enhancement by adopting the chaotic system for wavelet tree based digital image watermarking," in Proceedings of the 16th IEEE International Conference on Image Processing (ICIP '09), pp. 3661-3664, IEEE, November 2009.

[22] R.-S. Run, S.-J. Horng, W.-H. Lin, T.-W. Kao, P. Fan, and M. K. Khan, "An efficient wavelet-tree-based watermarking method," Expert Systems with Applications, vol. 38, no. 12, pp. 1435714366, 2011.

[23] F. Davoine, "Comparison of two wavelet based image watermarking schemes," in Proceedings of the International Conference on Image Processing (ICIP '00), vol. 3, pp. 682-685, IEEE, Vancouver, Canada, September 2000.

[24] G. Zhang, S. Wang, and Q. Wen, "An adaptive block-based blind watermarking algorithm," in Proceedings of the 7th International Conference on Signal Processing (ICSP '04), pp. 2294-2297, IEEE, August 2004.

[25] F. Khelifi, A. Bouridane, F. Kurugollu, and A. I. Thompson, "An improved wavelet-based image watermarking technique," in Proceedings of the IEEE Conference on Advanced Video and Signal Based Surveillance (AVSS '05), pp. 588-592, IEEE, Como, Italy, 2005. 
[26] W.-H. Lin, S.-J. Horng, T.-W. Kao, P. Fan, C.-L. Lee, and Y. Pan, "An efficient watermarking method based on significant difference of wavelet coefficient quantization," IEEE Transactions on Multimedia, vol. 10, no. 5, pp. 746-757, 2008.

[27] W.-H. Lin, Y.-R. Wang, S.-J. Horng, T.-W. Kao, and Y. Pan, "A blind watermarking method using maximum wavelet coefficient quantization," Expert Systems with Applications, vol. 36, no. 9, pp. 11509-11516, 2009.

[28] Y.-R. Wang, W.-H. Lin, and L. Yang, "An intelligent watermarking method based on particle swarm optimization," Expert Systems with Applications, vol. 38, no. 7, pp. 8024-8029, 2011.

[29] V. S. Verma and R. K. Jha, "Improved watermarking technique based on significant difference of lifting wavelet coefficients," Signal, Image and Video Processing, 2014.

[30] H.-H. Tsai, Y.-J. Jhuang, and Y.-S. Lai, "An SVD-based image watermarking in wavelet domain using SVR and PSO," Applied Soft Computing, vol. 12, no. 8, pp. 2442-2453, 2012.

[31] M. J. Sahraee and S. Ghofrani, "A robust blind watermarking method using quantization of distance between wavelet coefficients," Signal, Image and Video Processing, vol. 7, no. 4, pp. 799807, 2013.

[32] B. Ma, Y. Wang, C. Li, Z. Zhang, and D. Huang, "A robust watermarking scheme based on dual quantization of wavelet significant difference," in Proceedings of the Pacific-Rim Conference on Multimedia (PCM '12), 2012.

[33] P. Meerwald, C. Koidl, and A. Uhl, "Attack on watermarking method based on significant difference of wavelet coefficient quantization," IEEE Transactions on Multimedia, vol. 11, no. 5, pp. 1037-1041, 2009.

[34] J. Zhang, L. Tian, and H.-M. Tai, "A new watermarking method based on chaotic maps," in Proceedings of the IEEE International Conference on Multimedia and Expo (ICME '04), vol. 2, pp. 939942, IEEE, Taipei, Taiwan, June 2004.

[35] B. Chen and G. W. Wornell, "Quantization index modulation: a class of provably good methods for digital watermarking and information embedding," IEEE Transactions on Information Theory, vol. 47, no. 4, pp. 1423-1443, 2001.

[36] The USI-SIPI image database, volume 3, http://sipi.usc.edu/ database/. 

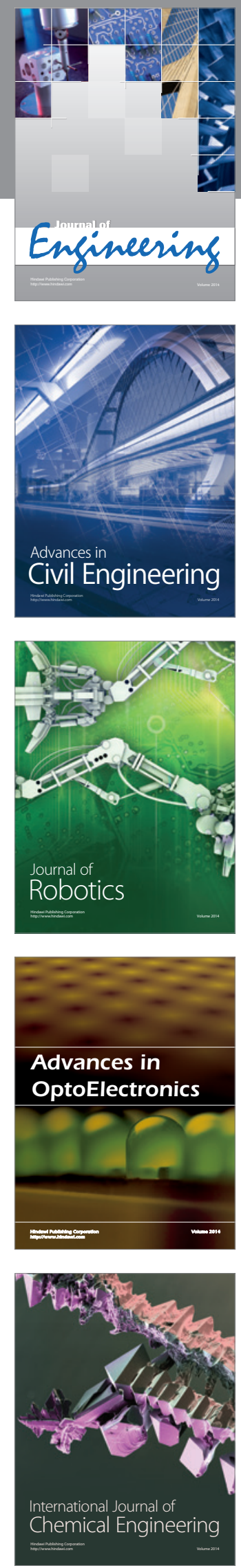

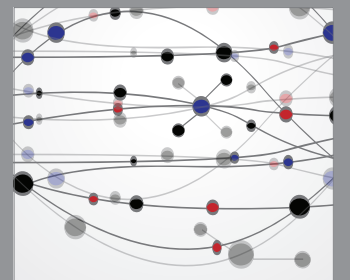

The Scientific World Journal
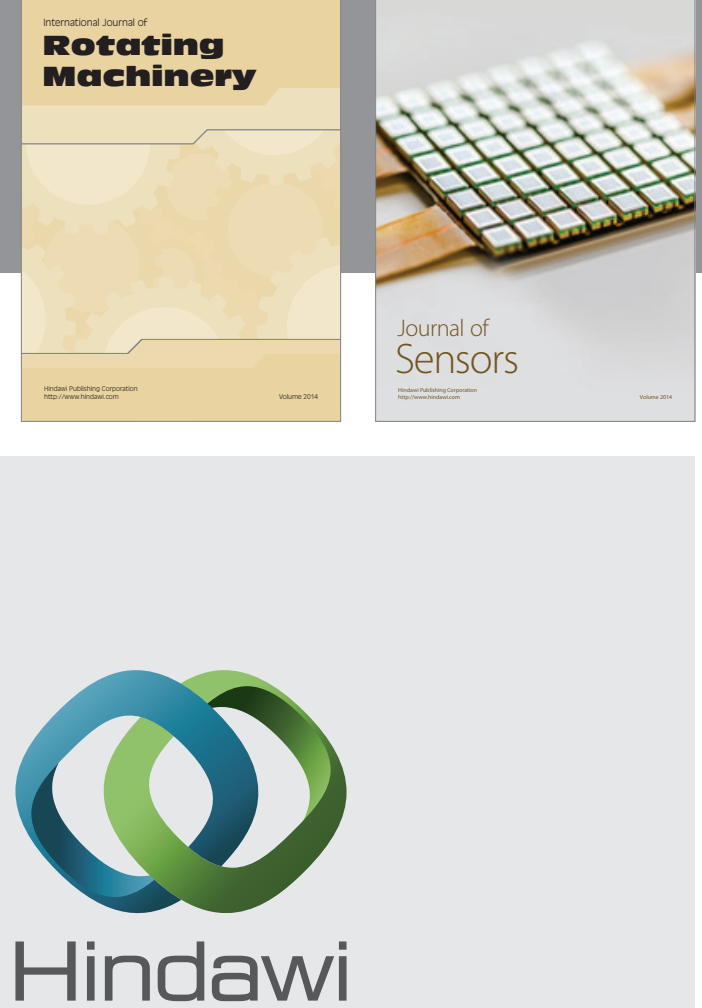

Submit your manuscripts at http://www.hindawi.com
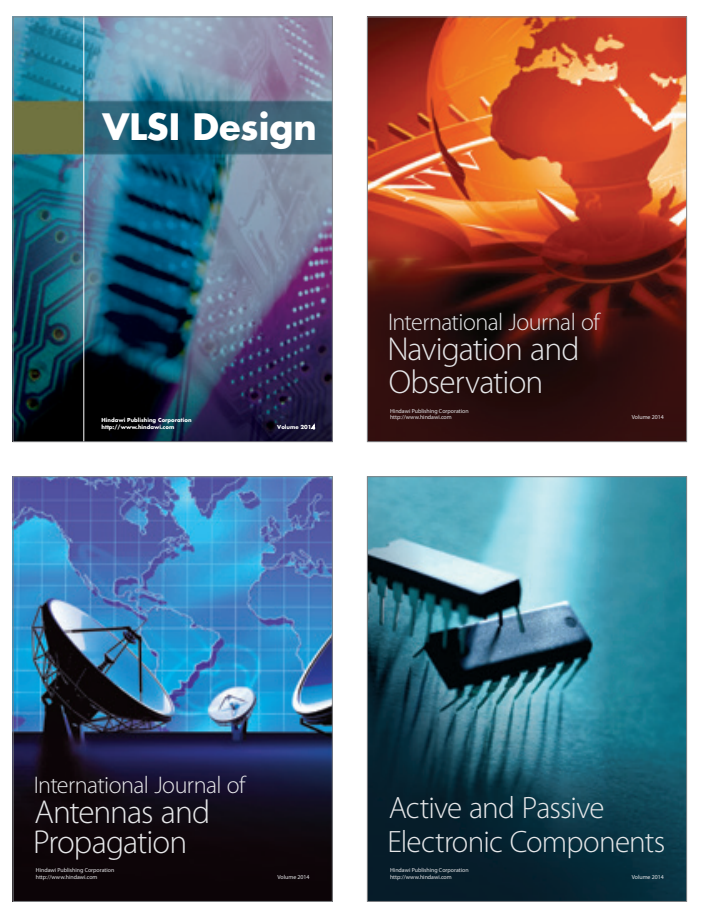
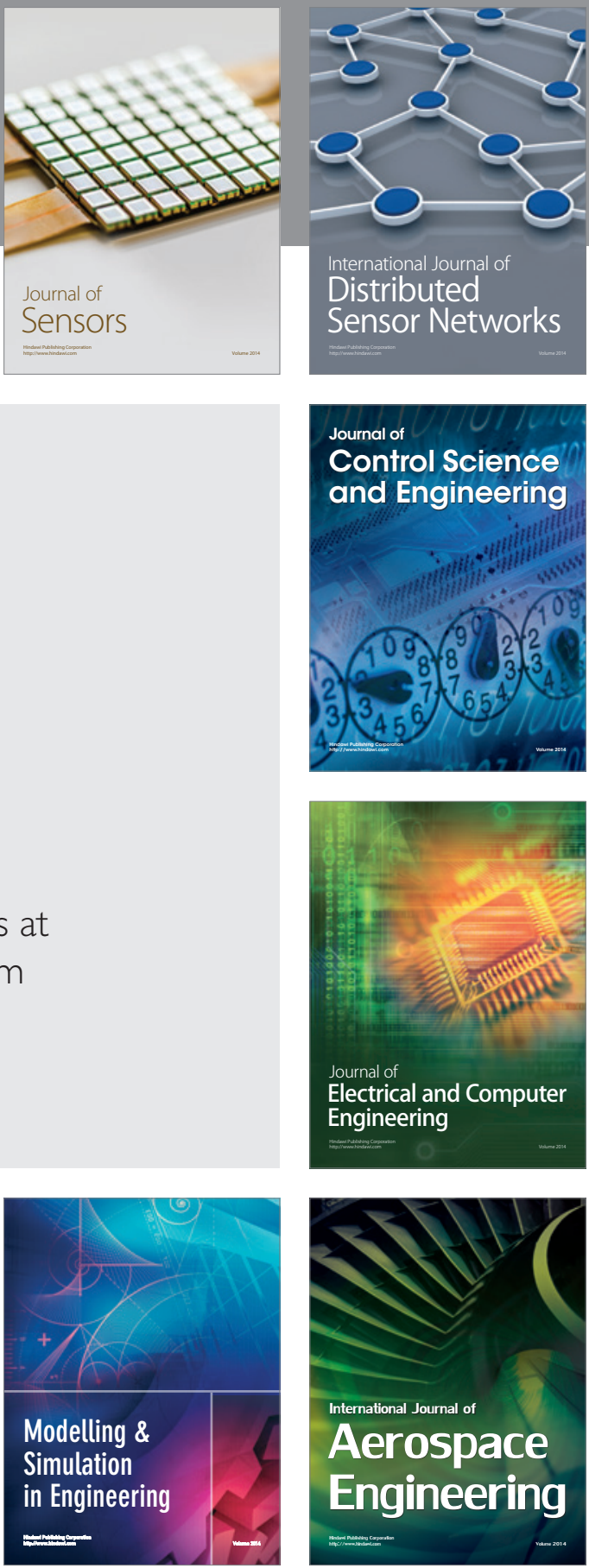

Journal of

Control Science

and Engineering
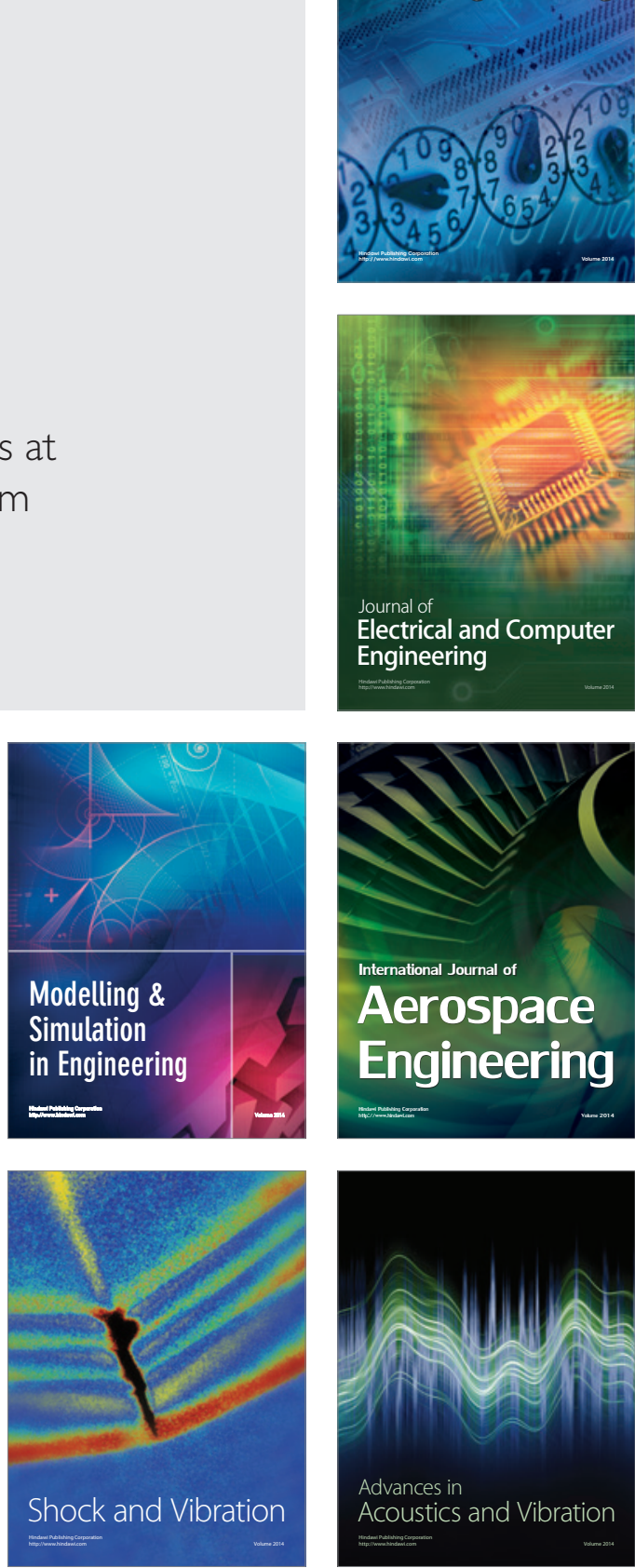